\title{
Acute pancreatitis in childhood-onset systemic lupus erythematosus: Case report
}

\author{
Yu-feng Li, M.D. Ph.D. ${ }^{a}$ and Min-jiang Wei, M.D. ${ }^{a}$
}

\begin{abstract}
Systemic lupus erythematosus (SLE) is a multisystem disease with protean manifestations. Lupus pancreatitis is the second most common disease associated with SLE-related acute abdomen. Although acute pancreatitis is rare it is clinically important because this condition can be life threatening if not treated promptly. Here, we report a case of a 10-year-old girl who developed SLE-associated pancreatitis after steroids therapy that was subsequently complicated by fungal septicaemia. Her clinical symptoms and signs markedly improved after administration of glucocorticoids and cyclophosphamide.

Keywords: systemiclupus ery thematosus; acutepancreatitis; fungal infection; septicaemia; child.
\end{abstract}

http: / / dx.doi.org/10.5546/ aap.2019.eng.e279

To cite: $\mathrm{Li}$ Y, Wei M. Acute pancreatitis in childhood-onset systemic lupus erythematosus: Case report. Arch Argent Pediatr 2019;117(3):e279-e283.

\section{INTRODUCTION}

Systemic lupus erythematosus (SLE) is a multisystem disease with protean manifestations. Abdominal pain is a common symptom in patients with SLE-related gastrointestinal involvement. ${ }^{1}$ Lupus pancreatitis is the second most common disease associated with SLE-related acute abdomen ${ }^{2}$. Gastrointestinal involvement is observed in $19.4 \%$ of paediatric SLE patients, whereas SLE-related pancreatitis occurs in $6.0 \%$ of paediatric SLE patients ${ }^{3}$. Although acute pancreatitis is rare, ${ }^{4,5}$ it is clinically important because this condition can be life threatening if not treated promptly. Here, we report a case of a young girl who developed SLE-associated pancreatitis that was subsequently complicated by fungal septicaemia.

a Department of Pediatric Nephrology, Xinhua Hospital Affiliated to Shanghai Jiaotong University School of Medicine, Shanghai 200092, China.

E-mail address:

Min-jiang Wei, M.D. Ph.D.: wmj10@12-6.com.cn

Funding: None.

Conflict of interest: None.

Received: 24-7-2018

Accepted: 10-12-2018

\section{CASE}

A 10-year-old girl presented first with oedema of the eyelids, gingival ulcers and continuous fever for more than 7 days. No history of photosensitivity. The liver and spleen were palpable $4 \mathrm{~cm}$ and $2 \mathrm{~cm}$, respectively, below the right and left costophrenic margins, respectively. No joint swelling or tenderness on extremities was noted. A neurological examination revealed no abnormalities. During the hospitalization, initial laboratory investigations produced the following findings: haemoglobin $127 \mathrm{~g} / \mathrm{L}$, white cell count $1.85 \times 10^{9} / \mathrm{L}$ (neutrophils $59.8 \%$, lymphocytes $33.3 \%$, monocytes $5 \%$ ), platelet count $100 \times 10^{9} / \mathrm{L}$, erythrocyte sedimentation rate $20 \mathrm{~mm} /$ hour, and C-reactive protein less than $8 \mathrm{mg} / \mathrm{L}$. The following results were obtained in liver function analyses: alanine aminotransferase $48 \mathrm{U} / \mathrm{L}$ (normal range $0-75 \mathrm{U} / \mathrm{L}$ ), aspartate aminotransferase $82 \mathrm{U} / \mathrm{L}$ (normal range 8-38 U/L), total protein $47.7 \mathrm{~g} / \mathrm{L}$ (normal range 63-82 g/L), albumin $21.2 \mathrm{~g} / \mathrm{L}$ (normal range 35-50 g/L), total bilirubin $2.4 \mu \mathrm{mol} / \mathrm{L}$, triglyceride $2.54 \mathrm{mmol} / \mathrm{L}$, alkaline phosphatase $119 \mathrm{U} / \mathrm{L}$, amylase $87 \mathrm{U} / \mathrm{L}$ (normal: 30-110 U/L), lipase 146 U/L (normal: 23$300 \mathrm{U} / \mathrm{L}$ ), and $\gamma$-GTP $14 \mathrm{U} / \mathrm{L}$. Kidney function analyses indicated that blood urea nitrogen was $8.98 \mathrm{mmol} / \mathrm{L}$ (normal range 2.86-7.14 mmol/L) and that creatinine was $66 \mu \mathrm{mol} / \mathrm{L}$ (normal range 35-97 $\mu \mathrm{mol} / \mathrm{L}$ ). Urinalysis revealed proteinuria, with a red blood cell count of 8-10/high-power field. The patient's 24-hour proteinuria excretion was $3.06 \mathrm{~g} /$ day. Immunological studies produced the following results: antinuclear antibody 1:640, anti-dsDNA antibody positive, p-ANCA antibody positive, anti-Sm antibody negative, anti-SS-A antibody and anti-SS-B antibody negative. The direct Coombs test was positive. Concentrations of complement components were extremely low: CH50 2.2 U/L (normal range 10-65 U/L), C3 $0.18 \mathrm{~g} / \mathrm{L}$ (normal range 0.9-1.8 g/L) and C4 $0.06 \mathrm{~g} / \mathrm{L}$ (normal range 0.1-0.4 g/L). A chest roentgenogram revealed no abnormalities.

Five days after hospitalization, a sudden seizure occurred. Features of seizure were consciousness loss, trismus, cyanosis, generalized tonic-clonic seizure without fever. Head CT 
scan and cerebrospinal fluid (CSF) examination were carried out immediately. CT scan and CSF examination were all normal. Her seizure was due to hyponatremia (serum sodium $120 \mathrm{mmol} / \mathrm{L}$ ) or hypocalcemia (serum calcium $1.56 \mathrm{mmol} / \mathrm{L}$ ). The patient was admitted to the intensive care unit. Haemoglobin and white cell count were decreased to $94 \mathrm{~g} / \mathrm{L}$ and $0.36 \times 10^{9} / \mathrm{L}$, respectively. Computed tomography (CT) revealed pleural effusion and ascites. The patient was diagnosed with SLE according to the diagnostic criteria of the American College of Rheumatology. The patient's condition did not permit renal biopsy. After the diagnosis was obtained, methylprednisolone pulse therapy $(20 \mathrm{mg} / \mathrm{kg}$ body weight intravenously for three consecutive days) was administered beginning on day 7 of hospitalization followed by oral prednisone ( $2 \mathrm{mg} / \mathrm{kg}$ per day) and high-dose immunoglobulin ( $1 \mathrm{~g} / \mathrm{kg}$ body weight intravenously for two consecutive days). She developed acute pancreatitis with severe abdominal pain, pleural effusion and ascites. Blood analysis revealed increased serum amylase (192 U/L) and lipase (726 U/L) levels and increased CRP (33 mg/L). Ultrasound study (US) of the abdomen revealed a markedly enlarged pancreas without stones in the gallbladder. The common bile duct was not dilated. CT of the abdomen revealed a swollen gallbladder with pericholecystic oedema and diffuse enlargement and homogeneous enhancement of the pancreas, spleen and liver. An endoscopic retrograde cholangiopancreatogram (ERCP) indicated diffuse narrowing of the common pancreatic duct with multiple strictures.

Another three pulses of methylprednisolone were administered, and immunosuppression with cyclosporine $(4.5 \mathrm{mg} / \mathrm{kg}$ per day) was added. On day 29 of hospitalization, blood culture results revealed fungal septicaemia (Candida parapsilosis). Voriconazole $(7 \mathrm{mg} / \mathrm{kg}$ per day) was added to treat this fungal septicaemia. Candida parapsilosis septicaemia relapsed several times during her hospitalization. Peripherally Inserted Central Catheter (PICC)-related bloodstream infection was considered. The fungal septicaemia was controled after PICC removal. The girl failed to respond of treatment with glucocorticoids plus cyclosporine. Urinalysis revealed massive proteinuria ( $15.14 \mathrm{~g} /$ day). The patient's immunosuppressive treatment was switched to oral mycophenolate mofetil (34 mg/ $\mathrm{kg}$ per day). However, 24-hour urine collection results remained greater than $10 \mathrm{~g} /$ day. On day 113 of hospitalization, cyclophosphamide pulse therapy was therefore administered (8-12 mg/ kg/ day for two consecutive days every 2 weeks). Mycophenolate mofetil was tapered down after cyclophosphamide administration, and corticosteroids were tapered to $1.5 \mathrm{mg} / \mathrm{kg}$ per day. Histological study of a needle biopsy specimen of the kidney revealed diffuse proliferative lupus nephritis of class III and class $\mathrm{V}$ according to the International Society of Nephrology / Renal Pathology Society (ISN/RPS) 2003 classification of lupus nephritis ${ }^{6}$. After four courses of cyclophosphamide pulses, the patient's 24-hour urine excretion was reduced to $1732 \mathrm{mg}$, and all laboratory test results had improved. During her hospitalization, the patient did not receive anything by mouth and was administered intravenous fluids and total parenteral nutrition until her abdominal pain had disappeared and her pancreatic enzymes had normalized. After 177 days of hospitalization the patient was discharged (Figure 1). Although brain magnetic resonance imaging revealed signs of diffuse atrophy on day 42 after discharge, she has been free of symptoms for a five-year period. The patient is now asymptomatic with low-dose $(2.5 \mathrm{mg}$ alternate day oral) oral steroid intake and being followed up in our outpatient unit.

\section{DISCUSSION}

SLE is a multisystem disease with the potential to involve almost every system and organ in the body. The prevalence of SLE-related pancreatitis varies from 0.67 to $4 \%$ in SLE patients. ${ }^{7}$ The annual incidence of SLE-related pancreatitis is estimated to be 0.4-1.1 per 1000 lupus patients. ${ }^{8}$ The prevalence of such pancreatitis is higher for paediatric-onset SLE $(5.22 \%)$ than for adult-onset SLE. ${ }^{9}$ Approximately $91 \%$ of pancreatitis cases diagnosed in patients with paediatric-onset SLE involve acute pancreatitis; in contrast, only $9 \%$ of such cases involve acute recurrent pancreatitis. ${ }^{10}$

The aetiology of pancreatitis in SLE remains unclear. Common causes of lupus pancreatitis have been defined as the mechanical obstruction of the pancreatic duct (most frequently as a result of choledocholithiasis) and toxicmetabolic causes (secondary to alcohol intake, treatment with certain drugs such as steroids, hyperkalaemia, or hypertriglyceridaemia). Steroid therapy was unlikely to be the cause of the pancreatitis observed in the described patient, as an additional, higher-dose steroid treatment 
FIGURE1. Laboratory results during treatment of pancreatitis episodes
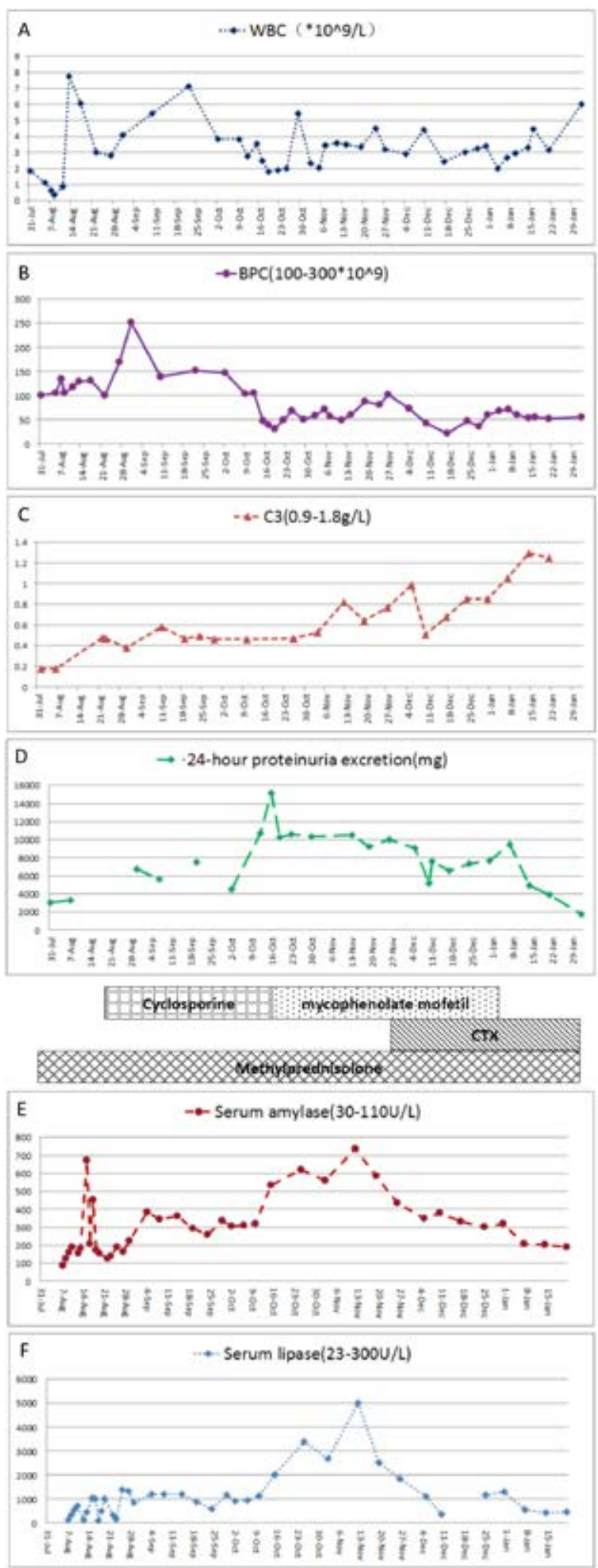

Cyclosporine: oral $4.5 \mathrm{mg} / \mathrm{kg}$ per day for 7 weeks; Mycophenolate mofetil: oral 34mg/ $\mathrm{kg}$ per day for 10 weeks; Cyclophosphamide: intravenous infusion cyclophosphamide $8-12 \mathrm{mg} / \mathrm{kg} /$ day for two consecutive days every 2 weeks; Methylprednisolone: oral methyprednisone $1.6 \mathrm{mg} / \mathrm{kg}$ per day for 8 weeks then $1.2 \mathrm{mg} / \mathrm{kg}$ per day. 
resulted in relief of her signs and symptoms. In this case, after excluding other common causes of acute pancreatitis, we diagnosed the patient with SLE with acute pancreatitis. Imaging studies, particularly abdominal CT scans, are helpful for diagnosing certain SLE-related gastroenteropathies. CT is recommended for evaluating the severity of pancreatitis. ${ }^{11}$

Lupus pancreatitis has been defined as the presence of typical clinical symptoms (including abdominal pain, nausea, and vomiting), with confirmation via a greater than 3 -fold increase in serum amylase or lipase or evidence from imaging findings (CT or ultrasonography) or endoscopic retrograde cholangiopancreatography. ${ }^{7}$ The following diagnostic criteria for autoimmune pancreatitis were proposed by the Japan Pancreatic Society in 2002: 1) pancreatic imaging studies reveal diffuse narrowing of the main pancreatic duct with irregular walls and diffuse enlargement of the pancreas; 2 ) laboratory data indicate abnormally elevated levels of serum gamma-globulin and/or IgG or the presence of autoantibodies; and 3) histopathological examination of the pancreas reveals fibrotic changes with lymphocyte and plasma cell infiltration. ${ }^{12}$

Corticosteroids should be used for the medical management of SLE-related acute pancreatitis; in particular, treatment with additional steroids at high doses has been successful in the management of SLE-related pancreatitis., ${ }^{2,13}$ In this report, the patient developed SLE-related acute pancreatitis concurrently with lupus nephritis; both complications were successfully treated with corticosteroids. Immunosuppressive agents such as mycophenolate mofetil or cyclophosphamide can be used in combination with corticosteroids. Notably, supportive measures were also extremely important. The described patient exhibited heavy proteinuria and hypoalbuminaemia, and her nutritional support was completely obtained from total parenteral nutrition by PICC. Good supportive measures are helpful for facilitating functional recovery and improving patient outcomes ${ }^{2}$. In severe cases, plasmapheresis and intravenous gamma-globulin infusion may be beneficial ${ }^{13}$. Our case suggests that methylprednisolone pulse therapy should be promptly administered if clinical and biochemical investigation suggest that SLE is responsible for pancreatitis. Cyclophosphamide pulses should be the first line of immunosuppressive agents used for SLE-related pancreatitis. ${ }^{14}$ Early and aggressive treatment may save the patient's life.

\section{REFERENCES}

1. Li Z, Xu D, Wang Z, Wang Y, et al. Gastrointestinal system involvement in systemic lupus erythematosus. Lupus. 2017; 26(11):1127-38.

2. Tu YL, Yeh KW, Chen LC, Yao TC, et al. Differences in disease features between childhood-onset and adult-onset systemic lupus erythematosus patients presenting with acute abdominal pain. Semin Arthritis Rheum. 2011; 40(5):447-54.

3. Limwattana S, Dissaneewate P, Kritsaneepaiboon S, Dendumrongsup T, et al.Systemiclupus erythematosus-related pancreatitis in children. Clin Rheumatol. 2013; 32(6):913-8.

4. Xu D, Yang H, Lai CC, Li P, et al. Clinical analysis of systemic lupus erythematosus with gastrointestinal manifestations. Lupus. 2010; 19(7):866-9.

5. Alves SC, Fasano S, Isenberg DA. Autoimmune gastrointestinal complications in patients with systemic lupus erythematosus: case series and literature review. Lupus. 2016; 25(14):1509-19.

6. Weening JJ, D'Agati VD, Schwartz MM, Seshan S, et al. The classification of glomerulonephritis in systemic lupus erythematosus revisited. J Am Soc Nephrol. 2004; 15(2): 241-50.

7. Yang $\mathrm{Y}, \mathrm{Ye} \mathrm{Y}$, Liang L, Wu T, et al. Systemic-lupus-erythematosus-related acute pancreatitis: a cohort from South China. Clin Dev Immunol. 2012; 2012:568564.

8. Makol A, Petri M. Pancreatitis in systemic lupus erythematosus: frequency and associated factors - a review of the Hopkins Lupus Cohort. J Rheumatol. 2010; 37(2):341-5.

9. Wang CH, Yao TC, Huang YL, Ou LS, et al. Acute pancreatitis in pediatric and adult-onset systemiclupus erythematosus: a comparison and review of the literature. Lupus. 2011; 20(5):443-52.

10. Marques VL, Gormezano NW, Bonfá E, Aikawa NE, et al. Pancreatitis Subtypes Survey in 852 Childhood-Onset Systemic Lupus Erythematosus Patients. J Pediatr Gastroenterol Nutr. 2016; 62(2):328-34.

11. Chang YJ, Chao HC, Kong MS, Hsia SH, et al. Acute pancreatitis in children. Acta Paediatr. 2011; 100(5):740-4.

12. Members of the Criteria Committee for Autoimmune Pancreatitis of the Japan Pancreas Society. Diagnosis criteria for autoimmune pancreatitis by the Japan Pancreas Society. J Jpn Pan Soc. 2002; 17:585-7.

13. Wang Q, Shen M, Leng X, Zeng X, et al. Prevalence, severity, and clinical features of acute and chronic pancreatitis in patients with systemic lupus erythematosus. Rheumatol Int. 2016; 36(10):1413-9.

14. Perrin L, Giurgea I, Baudet-Bonneville V, Deschênes G, et al. Acute pancreatitis in paediatric systemic lupus erythematosus. Acta Paediatr. 2006; 95(1):121-4. 